\title{
Rastreamento do câncer de mama no Brasil: ainda há tempo para refletirmos
}

\section{Breast cancer screening in Braqil: there is still time to rethink}

Segundo estimativas do Instituto Nacional do Câncer (INCA), no Brasil, em 2012, tivemos 52.333 casos novos do câncer de mama. Não existem informações seguras sobre o estadiamento destas pacientes e a taxa bruta de mortalidade pela doença, fazendo com que a reflexão e o planejamento estratégico do rastreamento da doença sejam ações pouco efetivas ${ }^{1}$.

Em 2009, a Fundação Oncocentro do Estado de São Paulo (FOSP) reuniu dados obtidos do Registro Hospitalar de Câncer (RHC) de 42 Centros de Alta Complexidade em Oncologia (CACON), no estado de São Paulo. Foram registrados 194.918 casos de câncer de 2000 a 2005, sendo 27.023 de câncer de mama no sexo feminino $(27,2 \%)$. Informações sobre idade e estadiamento foram obtidas de 26.076 casos $^{2}$. A distribuição dos estádios em relação às faixas etárias está expressa na Tabela 1.

Ao analisarmos os dados da Tabela 1, foi possível observar que existe um predomínio do diagnósticos de câncer de mama em estádios avançados no estado de São Paulo. A taxa de diagnóstico da doença nos estádios IIb (que corresponde à mulheres com tumores maiores que $5 \mathrm{~cm}$ ou menores mas com axila clinicamente positiva); III (tumores localmente avançados) e IV (doença metastática) alcança 51,2\%. Este cenário é altamente discrepante se a casuística de países desenvolvidos for analisada. Segundo dados do Surveillance Epidemiology, and End Results (SEER), do National Cancer Institute nos Estados Unidos, entre 302.763 casos registrados de 1988 a 2001, somente 5,6\% deles foram diagnosticados no estádio III, 50,6\% como 0 e I e 50,3\% com tumores menores que $20 \mathrm{~mm}$ (T1) ${ }^{3}$. No Reino Unido,

Tabela 1. Distribuição dos casos de câncer de mama no Estado de São Paulo em função do estadiamento clínico e faixa etária, segundo dados do Registro Hospitalar de Câncer (Fundação Oncocentro), no período de 2000 a 2005

\begin{tabular}{lccccc}
\hline Estadiamento clínico & $<40$ anos (\%) & $40-49(\%)$ & $50-69(\%)$ & $>69(\%)$ & Total (\%) \\
\hline 0 & 4,2 & 7,9 & 6,7 & 3,9 & 6,2 \\
I & 12,1 & 17,4 & 18,8 & 17,3 & 17,4 \\
IIla & 24,8 & 23,4 & 25,7 & 25,3 & 25,1 \\
IIb & 18,7 & 17,1 & 14,5 & 14,6 & 15,6 \\
III & 30,3 & 26,5 & 25,9 & 28,6 & 27,0 \\
IV & 9,9 & 7,1 & 8,4 & 10,3 & 8,5 \\
\hline
\end{tabular}

Correspondência

Daniel Guimarães Tiezzi Avenida Bandeirantes, 3.900 CEP: $14049-900$

Ribeir̃̃o Preto (SP), Brasil

Recebido

$15 / 08 / 2013$

'Departamento de Ginecologia e Obstetrícia da Faculdade de Medicina de Ribeirão Preto, Universidade de São Paulo - USP; Divisão de Mastologia e Ginecologia Oncológica do Hospital das Clínicas de Ribeirão Preto, Universidade de São Paulo - USP - Ribeirão Preto (SP), Brasil.

Conflito de interesses: não há. 
onde o sistema de saúde é público, 59,6\% das ocorrências de câncer de mama são diagnosticadas como T1 $1^{4}$. Analisando todos os registros no RHC, entre 2000 a 2012 no estado de São Paulo (61.086 casos), observou-se que 33,7\% dos casos foram diagnosticados com tumores menores do que $20 \mathrm{~mm}$ de diâmetro (T1).

O relatório da FOSP também expõe os dados de sobrevida global da população com câncer de mama em função do estadiamento. A taxa de sobrevida global em cinco anos foi de $98 \%$ para o estádio clínico (EC) I, $90 \%$ para o EC IIa, $82 \%$ para o EC IIb, $60 \%$ para o EC III e de $30 \%$ para o EC IV. Naquele período, foram registradas um total de 1.427 mortes nos estádios I e IIa e 4.906 mortes nos estádios IIb, III e IV. O estádio IIa é clinicamente evidente na grande maioria dos casos. De uma forma hipotética, se todas as mulheres com câncer de mama nos estádios IIb, III e IV fossem diagnosticadas com a doença clinicamente evidente em estágio IIa, nós teríamos prevenido a morte de 3.570 mulheres naquele período. No Reino Unido, $48 \%$ dos casos sintomáticos são diagnosticados com tumores menores de $20 \mathrm{~mm}$ e apenas $7 \%$ são maiores que $5 \mathrm{~cm}^{4}$. Esta observação sustenta a possibilidade de diagnóstico em estádios menos avançados, por meio de informação e busca ativa.

Segundo o Censo 2000 (IBGE) , no Estado de São Paulo, 4.537.642 mulheres estavam dentro da faixa etária para a qual se preconiza o rastreamento com mamografia (2,4 milhões de 40-49 anos e 2,1 milhões de 50-69 anos), ou seja, se fosse realizada uma mamografia a cada dois anos neste grupo de pacientes, seriam necessários 11.3 milhões de exames nos cinco anos correspondentes ao levantamento realizado pela FOSP. Naquele período, o custo direto de cada mamografia era de $\mathrm{R} \$ 30,00$, de acordo com a tabela de procedimentos do Sistema Único de Saúde (SUS). O Governo deveria ter investido cerca de $\mathrm{R} \$ 340$ milhões para cobrir o rastreamento mamográfico somente na população do estado de São Paulo. Tal investimento não inclui os gastos referentes aos exames adicionais gerados pelas mamografias BI-RADS zero; os custos referentes aos procedimentos de diagnóstico e os gastos indiretos com o transporte e o afastamento da mulher de suas atividades profissionais para ser submetida ao rastreamento. Em 2003, o Governo Federal financiou 2.026.111 mamografias em todo o Brasil ${ }^{6}$. Em cinco anos, seriam cerca de 10 milhões de exames, número insuficiente para rastrear a população do estado de São Paulo que representava 21,8\% dos brasileiros em 2000.

O rastreamento com mamografia reduz o risco de morte pela doença na ordem de $20 \%$. Segundo a faixa etária, o decréscimo no risco de morte é de $15 \%$ para a população entre 40 a 49 anos (OR =0,85; IC95\% 0,75-0,96), 14\% entre 50 a 59 anos $\left(\mathrm{OR}=0,86\right.$; IC95\% 0,75-0,99) e 38\% para 60 a 69 anos $\left(\mathrm{OR}=0,68\right.$; IC95\% 0,54-0,87) ${ }^{7}$. Se a população do estado de São Paulo estivesse sendo submetida ao rastreamento adequado durante o período compreendido no levantamento da FOSP, aproximadamente 1.266 óbitos com o rastreamento teriam sido prevenidos, ou seja, metade do número de mortes que poderiam ter sido salvas com o diagnóstico clínico do câncer de mama em estádios menos avançados (IIa). Além disso, teríamos investido R \$268.000 para prevenir um óbito devido à doença.

O Ministério da Saúde (MS) publicou em 2004 um documento de consenso sobre o controle do câncer de mama no Brasil ${ }^{8}$. Segundo este, o rastreamento deve ser feito por meio do exame clínico da mama para todas as mulheres a partir dos 40 anos, sendo realizado anualmente. A mamografia de rastreamento deve ser oferecida às mulheres entre 50 a 69 anos (máximo de dois anos entre os exames), e todas devem ter garantia de acesso ao diagnóstico, tratamento e seguimento. Foi efetuado um levantamento do banco de dados da FOSP, baseando-se no RHC de 2009, com dados registrados cinco anos após a publicação do protocolo do MS. Naquele ano foram registrados 6.337 casos de câncer de mama e informações sobre o estadiamento podem ser obtidas de 6.187 deles. Observou-se o mesmo padrão de distribuição pelo EC do período de 2000 a 2005 (48,8\% das pacientes foram diagnosticadas nos estádios IIb, III e IV). Se analisarmos somente a faixa etária preconizada para o rastreamento com mamografia (50 a 69 anos), um total de $45,1 \%$ das mulheres foram diagnosticadas em estágios avançados. Os estádios 0 (carcinoma in situ) e I juntos perfazem $31,7 \%$ e o IIa, 22,9\%. Na Tabela 2 podemos comparar o estadiamento clínico das pacientes de 2000 a 2005 com o de 2009 .

Tabela 2. Distribuição dos casos de câncer de mama no Estado de São Paulo em função do estadiamento clínico, segundo dados do Registro Hospitalar de Câncer (Fundação Oncocentro), em dois períodos diferentes

\begin{tabular}{|c|c|c|}
\hline Estadiamento clínico & $2000-2005(\%)$ & $2009(\%)$ \\
\hline 0 & 6,5 & 8,3 \\
\hline I & 18,7 & 23,4 \\
\hline Ilb & 14,5 & 12,4 \\
\hline III & 25,9 & 25,5 \\
\hline
\end{tabular}


Analisando a Tabela 2, foi possível observar um discreto aumento nas taxas de detecção precoce do câncer de mama no estado de São Paulo. No entanto, esta diminuição não foi decorrente de redução no número de tumores localmente avançados, sugerindo que o rastreamento está atingindo a população mais bem orientada e que tem mais facilidade de acesso ao sistema de saúde. É evidente que ações de conscientização da população e investimento em treinamento do pessoal que atua nos níveis primários de assistência e identificação de mulheres sintomáticas (treinamento em exame físico das mamas) podem ser cruciais à redução do número de casos avançados e consequente decréscimo substancial da morte pela doença no Brasil. Vale ressaltar que tais medidas diminuíram os custos do tratamento primário da doença, bem como o investimento no tratamento paliativo de pacientes com doença metastática.

Em 1991, o governo brasileiro instituiu o Programa Agentes Comunitários em Saúde (PACS) que foi o precursor do Programa de Saúde da Família (PSF), implementado em 1994. As principais funções dos agentes de saúde são: levar à população informações capazes de promover o trabalho em equipe; visita domiciliar; planejamento das ações de saúde; promoção da saúde; prevenção e monitoramento das situações de risco e do meio ambiente; prevenção e monitoramento de grupos específicos; prevenção e monitoramento das doenças prevalentes e acompanhamento e avaliação das ações de saúde. Esse programa visa priorizar a assistência à saúde de famílias carentes ${ }^{9}$. Em 2009, os agentes de saúde do PSF e do PACS realizaram, em conjunto, cerca de 37 milhões de visitas domiciliares no estado de São Paulo ${ }^{10}$. Esses dados sugerem que o programa é abrangente. No entanto, comparando os dados de incidência do câncer de mama localmente avançado com o número de visitas domiciliares pelos agentes de saúde, pode-se imaginar que as equipes de saúde não estão treinadas e orientadas a identificarem mulheres com tal doença.

A análise dos dados expostos permite inferir que o programa de rastreamento do câncer de mama no Brasil é ineficaz. Provavelmente, ele é baseado no diagnóstico oportunístico e não tem uma abrangência satisfatória. As altas taxas de diagnóstico em estádios avançados sugerem que devemos investir de forma imediata na conscientização da população, utilizando a estrutura do PSF e PACS para este fim. A estrutura já existe e aparentemente deve ser bastante ativa, o que falta é o treinamento dos profissionais a fim de orientar a detecção precoce, por meio do autoexame das mamas; identificar pacientes em estádios iniciais; facilitar e agilizar o encaminhamento de pacientes com suspeita clínica a unidades de diagnóstico e tratamento do câncer (CACONs). O investimento na detecção precoce de casos sintomáticos parece ser urgente. É necessário primeiramente cuidar do grande número de mulheres sintomáticas, mas ainda sem doença avançada, para depois ser implementado um programa de detecção da doença subclínica.

\section{Referências}

1. Brasil. Instituto Nacional de Câncer José Alencar Gomes da Silva. Coordenação Geral de Ações Estratégicas. Coordenação de Prevenção e Vigilância [Internet]. Estimativa 2002: incidência de câncer no Brasil. Rio de Janeiro: INCA; 2011 . [citado 2013 Mar 6]. Disponível em: <http://www.inca.gov.br/estimativa/2012/>

2. São Paulo. Secretaria de Estado da Saúde. Fundação Oncocentro [Internet]. Sobrevida de pacientes com câncer no Estado de São Paulo: seis anos de seguimento pelo Registro Hospitalar de Câncer. 2009. Cadernos FOSP, 5. [citado 2013 Mar 6]. Disponível em: <http://www.fosp. saude.sp.gov.br/epidemiologia/docs/sobevida.pdf>

3. Ries LAG, Young JL, Keel GE, Eisner MP, Lin YD, Horner M, editors. SEER Survival Monograph: cancer survival among adults: U.S. SEER Program, 1988-2001, patient and tumor characteristics. Bethesda: National Cancer Institute; 2007. SEER Program, NIH Pub. No. 07-6215.

4. NHS Cancer Screening Program [Internet]. All breast cancer report: a UK analysis of all symptomatic and scree-detected breast cancer diagnosed in 2006. [cited 2012 Apr 12]. Available from: <http://www.cancerscreening.nhs.uk/breastscreen/all-breast-cancer-report.pdf>

5. Brasil. Instituto Brasileiro de Geografia e Estatística [Internet]. Censo 2000. [citado 2011 Jan 8]. Disponível em: <http://www.ibge.gov.br/ home/estatistica/populacao/default_censo_2000.shtm>

6. Brasil. Ministério da Saúde. Portal Saúde [Internet]. Em 2009 SUS financiou 3 milhões de mamografias. [citado 2011 Jan 8]. Disponível em: <http://portal.saude.gov.br/saude/visualizar_texto.cfm?idtxt=3389l>

7. Gregory KD, Sawaya GF. Updated recommendation for breast cancer screening. Curr Opin Obstet Gynecol. 2010;22(6):498-505.

8. Brasil. Ministério da Saúde. Instituto Nacional de Câncer [Internet]. Controle do câncer de mama: documento de consenso. Rio de Janeiro: INCA; 2004. [citado $2011 \mathrm{Fev}$ 17]. Disponível em: <http://www.inca.gov.br/publicacoes/consensointegra.pdf>

9. Brasil. Ministério da Saúde. Coordenação Geral de Desenvolvimento de Recursos Humanos para o SUS/ SPS/MS. Coordenação de Atenção Básica/SAS/MS. Diretrizes para elaboração de programas de qualificação e requalificação dos Agentes Comunitários de Saúde. Brasília (DF): Ministério da Saúde; 1999.

10. Brasil. Ministério da Saúde. DATASUS [Internet]. Informações de Saúde. Estatísticas vitais. Número de visitas: PSF: PACS: Sistema de Informação de Atenção Básica - Situação de Saúde - São Paulo. Brasília (DF): Ministério da Saúde; 2009. [citado 201 1 Fev 17]. Disponível em: <http:// tabnet.datasus.gov.br/cgi/tabcgi.exe?siab/cnv/SIABSsp.def> 